cyclizine hydrochloride, which could have slowed his intestinal transit rate. Systemic toxicity is an alternative possibility. In view of the animal studies small bowel perforation is not an unexpected side effect, especially in association with the high dose of diclofenac sodium that this man was taking.

Non-steroidal anti-inflammatory drugs are often prescribed for pain that is unremitting and difficult to control, but their therapeutic window is small. With high dose slow release preparations it is easy to reach harmful concentrations by taking relatively few tablets. Patients taking slow release preparations of non-steroidal anti-inflammatory drugs should be warned specifically of the dangers of increasing the dose beyond that recommended. The risk of localised toxicity may be increased when non-steroidal antiinflammatory preparations are taken in conjunction with opiates or their derivatives, which decrease intestinal motility.

1 Day TK. Intestinal perforation associated with osmotic slow release indomethacin capsules. Br Med F 1983;287:1671-2.

2 Langman MJS, Morgan L, Worrall A. Use of anti-inflammatory drugs by patients admitted with small or large bowel perforaions and haemorrhage. BrMed f 1985;290:347-9.

3 Kent TH, Cardelli RM, Stander FW. Small intestinal ulcers and intestinal flora in rats given indomethacin. Am $\mathcal{J}$ Pathol 1969;54:237-45

4 Wax J, Cliger WA, Varner P, Bass P, Winder W. Relationship of the enterohepatic cycle to ulcerogenesis in the rat small bowel with flufenamic acid. Gastroenterology 1970;58:772-80.

5 Robert A. An intestinal disease produced experimentally by a prostaglandin deficiency. Gastroenterology 1975;69:1045-7.

\section{Unilateral deafness as a complication of the mumps, measles, and rubella vaccination}

Drs J Nabe-Nielsen and B Walter (Audiological Department, Aalborg Hospital, DK-9000 Aalborg, Denmark) write: A 7 year old girl, who had been found to have normal hearing by pure tone audiometry two years earlier, received a mumps, measles, and rubella vaccination without any immediate complications. Eleven days later she had a slight fever which lasted for two days. She then complained that she was deaf in her left ear. She had no dizziness, headaches, or earaches.

One month after the vaccination an ear, nose, and throat specialist found that she had a profound hearing impairment in her left ear by pure tone audiometry.

Ten weeks after the vaccination she had no complaints apart from the hearing loss. Pure tone audiometry showed anacusis in her left ear. In her right ear the hearing level was $0 \mathrm{~dB}$, with normal speech discrimination. No contralateral stapedius reflexes could be elicited from either ear. Ipsilateral stapedius reflexes were present in the right ear. Tympanometry was normal in both ears. Examination findings of the ears, nose, and throat were otherwise normal. Caloric testing showed no response in either ear to warm water and only weak but equal response to cold water. Postrotatory nystagmus was normal.

Eleven weeks after the mumps, measles, and rubella vaccination IgG antibodies to the mumps virus were found. Immunoglobulins $M$ and $A$ could not be found. The girl had never had symptoms of mumps. Her hearing impairment has not changed during the past 11 months, and she still has no other complaints.

Mumps is usually a benign disease, but it is an important cause of unilateral hearing impairment in children. With the development of the combined mumps, measles, and rubella vaccine vaccination should become more widespread, so reducing the amount of unilateral hearing loss in children. Adverse reactions to mumps, measles, and rubella vaccination are mainly in the form of fever. There are few serious complications, and most are reversible.

The Health Department in Denmark knows of no other case of hearing impairment after mumps, measles, and rubella vaccination. Only two cases of unilateral deafness have been reported before as a result of mumps vaccination. ${ }^{23}$

In our patient it seems reasonable to believe that the unilateral anacusis was caused by the mumps component of the mumps, measles, and rubella vaccine. This seems to be a rare complication, but it is important to be aware of this possibility during the first years of the vaccination programme, as it is only in older children that we will be able to discover this side effect. When nearly all children over 3 years of age have been vaccinated the discovery of unilateral deafness will be delayed and the relation to the vaccine will be difficult to prove.

Peltola $\mathrm{H}$, Hejnonen OP. Frequency of true adverse reactions to measles mumps rubella vaccine. Lancet 1986; ; $939-42$.

2 Hayden GF, Preblud SR, Orenstein WA, Conrad JL. Curren status of mumps and mumps vaccine in the United States. Pediatrics 1978;62:965-8.

3 Healy CE. Mumps vaccine and nerve deafness. Am 7 Dis Child 1972;123:612.

\section{Points}

\section{ABC of Eyes: Cataracts}

Mr D P Choyce (Westcliff on Sea, Essex SS0 8LR) writes: Messrs A R Elkington and P T Khaw clearly explained the current treatment of cataracts (25 June, p 1787). It is good to read that routine lens implantation is now accepted without question in most cases. They may, however, have missed a golden opportunity to extol the virtues of secondary lens implantation. There must be at least 100000 patients in the United Kingdom who had their cataracts removed before primary lens implantation became accepted practice not much more than five years ago whose visual rehabilitation was inhibited by reliance on cataract spectacles or contact lenses, the limitations of which were exposed by Messrs Elkington and Khaw. Most of these patients no longer attend hospital eye clinics (unless something has gone wrong with their aphakic eye(s)) and are generally unaware that they have not missed the boat, so to speak, and that they too could enjoy all the advantages of pseudophakic vision if only they asked. The arrival a few years ago of viscosurgery, of which the most widely used example is sodium hyaluronate, has rendered both primary and secondary lens implantation much safer. Perhaps this additional boon could have been briefly mentioned also.

Dr David GilberT (London SE21) writes: Messrs A R Elkington and P T Khaw (25 June, p 1787) stated that there is no effective medical treatment for established cataracts. This should be qualified with respect to central cataracts, in which, so long as the periphery of the lens remains clear, the use of a mydriatic agent can improve functional vision by increasing the ratio of focused to scattered light reaching the retina, particularly under bright ambient conditions. Accommodation and adaptation are, of course, lost, but these losses can be compensated for by appropriate spectacles such as photochromic trifocals. My own central cataracts have been managed in this way for over 10 years. It is a treatment option that is worth keeping in mind for the occasional patient who could benefit from it.

\section{Organs for transplantation}

Dr GREY E B Giddins (Royal National Orthopaedic Hospitals, Stanmore, Middlesex) writes: In response to Professor Geoffrey D Chisholm's article on organ transplantation (21 May, p 1419) several correspondents have pointed out the inaccuracy of his statement that "a patient not on a respirator can never be a donor" and have correctly stated that kidneys and corneas can be taken from patients who are not on respirators (2 July, p 68; 16 July, p 201). Sadly, once again, as on donor cards, there has been no mention of bones. Bone transplantation, which was first reported in $1908,{ }^{1}$ remains a neglected art in Britain especially when compared with that in North America, where most major orthopaedic centres have extensive transplant programmes. Bones can be harvested for fresh immediate use or delayed use almost whenever other organs are taken. Moreover, bone transplantation does not require cross matching, immunosuppres sion, or a "beating heart" donor, and only for fresh grafts is there a need for immediately available recipients. The uncommonness of bone harvesting in Britain represents a considerable waste of resources The inclusion of bones on donor cards may heighten both public and medical awareness and help to make better use of this facility.

1 Lexer E. Joint transplantation. Clinical Orthop 1985;197 4-10.

\section{Myocardial microthrombi in systemic} lupus erythematosus

Professor Priscilla Kincaid-Smith (Department of Medicine, Royal Melbourne Hospital, Victoria 3050 Australia) writes: The case reported by Dr J H Brown and others (28 May, p 1505) is similar to a series of 12 women with pregnancy related acute thrombotic microangiopathy and impaired renal function which we reported recently (in press). The common factor in these young women was the presence of a circulating lupus anticoagulant. Eight of the 10 women in whom the test was performed had a positive test for antiphospholipid (anticardiolipin) antibodies. These patients had fibrin thrombi in arteries, arterioles, and glomerular capillaries as seen in biopsy specimens. These findings are typical of thrombotic microangiopathy. Only two of 23 pregnancies in these women were successful, and pregnancy precipitated the acute thrombotic microangiopathy in most of our patients. One, however, had had a previous episode of thrombotic microangiopathy related to taking an ora contraceptive agent. This prompts the question whether the 22 year old patient described by the authors was using oral contraceptives before her fulminating fatal microangiopathy. [The authors confirm that the patient had never taken oral contraceptives and was not pregnant-ED.]

\section{Which patients should undergo laparoscopy?}

Dr Hamish MCA Foster (Hunter Oncology Centre, Waratah, New South Wales 2298, Australia) writes: agree with Mr S Patterson-Brown and others that a laparoscopy should be performed on all women who present with a clinical diagnosis of acute appendicitis (14 May, p 1363). The clinical diagnosis of acute appendicitis in women is difficult, especially in those of childbearing age. Accuracy rates can be as low as about a half. ${ }^{1}$ In the North Solomons province of Papua New Guinea laparoscopy has been used before operation in virtually all women with suspected appendicitis in an attempt to reduce the rate of unnecessary exploration and appendicectomy. Over three years 39 women with suspected appendicitis had a laparoscopy. Twenty nine required no further operation and were observed or received antibiotics for pelvic inflammatory disease. Eight had appendicectomies, and two required a laparotomy for pelvic disease. Unnecessary appendicectomy was thus avoided in 29 of the 39 patients ( $74 \%$ ), an important saving in a country where surgical resources are scarce. When the appendix could not be seen at laparoscopy an appendicectomy was carried out. When the appendix was normal and no other cause of the patient's abdominal pain could be found the patient was either observed in hospital or had an immediate appendicectomy. We have not found that laparoscopy is useful in men with suspected appendicitis in the North Solomons as most of them present with signs of late appendicitis (a mass or peritonitis). Laparoscopy is unnecessary and hazardous in such patients.

1 Chang FC, Hogle HH, Welling DR. The fate of the negative appendix. Am F Surg 1973;126:752-4.

\section{Undergraduate medical education}

Professor R M HARden (Centre for Medical Education, University of Dundee, Dundee DDl 9SY) writes: Dr Brian Jolly's letter about medical education in Britain (9 July, p 136) is misleading. His estimate of five educationalists working full time on medica educational problems in British medical schools is low. In this unit alone we have eight staff working full time in this subject. Dr Jolly's information about the lack of training programmes in medical education is equally out of date. In Dundee, in addition to three week courses in medical education, which have so far attracted more than 600 medical teachers from the United Kingdom and overseas, we now offer a distance learning diploma course in medical education. This was designed to meet the needs identified by Professor Peter Richards for "clinical teachers who understand the complexity of medicine to think about patterns of medical education for the future" (7 May, $\mathrm{p}$ 1279). The first two doctors have now completed the course successfully and 50 are enrolled currently. 